PROCEEDINGS OF THE AMERICAN MATHEMATICAL SOCIETY

Volume 126, Number 3, March 1998, Pages 791-800

S $0002-9939(98) 04387-1$

\title{
THE HAUSDORFF DIMENSION OF GRAPHS OF WEIERSTRASS FUNCTIONS
}

\author{
BRIAN R. HUNT
}

(Communicated by J. Marshall Ash)

\begin{abstract}
The Weierstrass nowhere differentiable function, and functions constructed from similar infinite series, have been studied often as examples of functions whose graph is a fractal. Though there is a simple formula for the Hausdorff dimension of the graph which is widely accepted, it has not been rigorously proved to hold. We prove that if arbitrary phases are included in each term of the summation for the Weierstrass function, the Hausdorff dimension of the graph of the function has the conjectured value for almost every sequence of phases. The argument extends to a much wider class of Weierstrass-like functions.
\end{abstract}

\section{INTRODUCTION}

Perhaps the most famous example of a continuous but nowhere differentiable function is that of Weierstrass,

$$
w(x)=\sum_{k=0}^{\infty} a^{k} \cos \left(2 \pi b^{k} x\right),
$$

where $0<a<1<b$, with $a b \geq 1$ (see [8]). Weierstrass proved that this function is nowhere differentiable for some of these values of $a$ and $b$, while Hardy [8] gave the first proof for all such $a$ and $b$. The graphs of this and related functions have often been studied as examples of fractal curves, first in [4] and more recently in [14], [3], and many of the other papers referred to here. The graph of $w(x)$ is roughly selfaffine, in the sense that $a w(b x)$ differs from $w(x)$ by the smooth function $\cos (2 \pi x)$, and this suggests the graph has dimension

$$
D=2+\frac{\log a}{\log b} .
$$

(Notice that $1<D<2$ provided $a b>1$.) Figure 1 shows the graph of $w(x)$ with $a=0.5$ and $b=3$; in this case $D=2-\log 2 / \log 3 \approx 1.37$.

The box-counting (capacity, entropic, fractal, Minkowski) dimension of the graph of $w$ was proved to be $D$ by Kaplan, Mallet-Paret, and Yorke [11], but it remains open whether the Hausdorff dimension of the graph of $w$ has the same value.

In this paper we consider the Weierstrass function with a random phase added to each term:

$$
w_{\Theta}(x)=\sum_{n=0}^{\infty} a^{n} \cos \left(2 \pi\left(b^{n} x+\theta_{n}\right)\right)
$$

Received by the editors September 3, 1996.

1991 Mathematics Subject Classification. Primary 28A80, 26A30, 28A78; Secondary 58F12. 


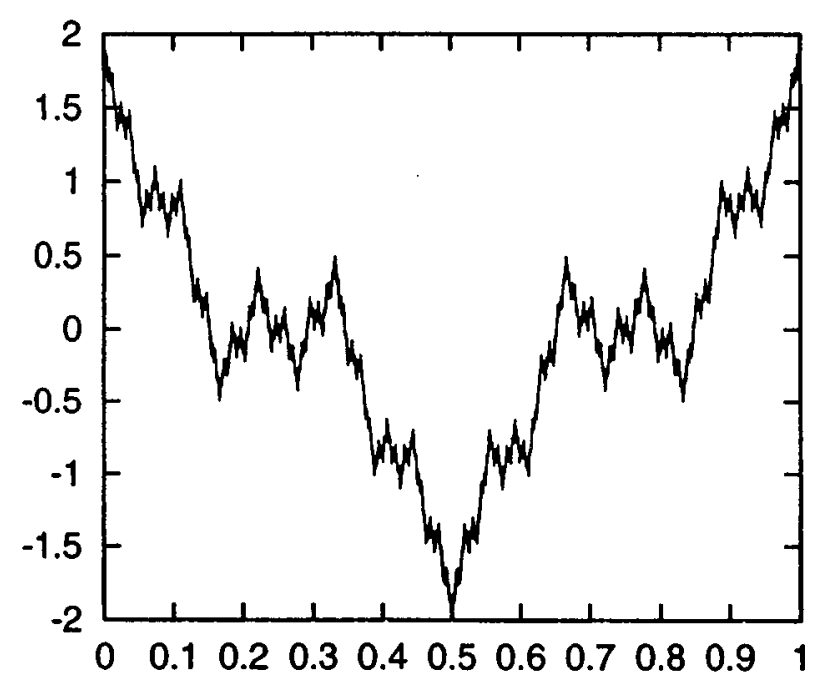

Figure 1. Graph of $w(x)$ with $a=0.5$ and $b=3$.

where $\Theta=\left\{\theta_{0}, \theta_{1}, \ldots\right\}$. We prove the following theorem.

Theorem 1. If each $\theta_{n}$ is chosen independently with respect to the uniform probability measure in $[0,1]$, then with probability one the Hausdorff dimension of the graph of $w_{\Theta}$ is $D$.

The proof is based on the potential-theoretic "energy" approach to Hausdorff dimension, which is reviewed in Section 2; this approach is also used in the heuristic argument given by Berry and Lewis [3] .

When $b$ is an integer, the graph of $w(x)$ arises as an invariant set for a smooth dynamical system $(x, y) \rightarrow T(x, y)$, where

$$
T(x, y)=\left(b x(\bmod 1), \frac{1}{a}(y-\cos (2 \pi x))\right) .
$$

Here $x$ lies on the circle obtained by identifying the endpoints of [0,1], and $y \in \mathbf{R}$. The graph of $y=w(x)$ can be thought of as a fractal basin boundary separating the initial conditions whose trajectories approach $y=-\infty$ under repeated application of $T$ from those whose trajectories approach $y=+\infty$; see for instance [1] and [10]. The graph can also be thought of as the attractor of the iterated function system generated by the $b$ branches of the inverse of $T$, as in [1]; see also [11] for a characterization of similar graphs as attractors.

The graph of $w_{\Theta}$ can be characterized as a fractal basin boundary for a random dynamical system. Let

$$
T_{\theta}(x, y)=\left(b x(\bmod 1), \frac{1}{a}(y-\cos (2 \pi(x+\theta)))\right),
$$

and consider the repeated application of $T_{\theta}$ with a new value of $\theta \in[0,1]$ chosen randomly at each iteration. Then if the sequence of random phases chosen is $\Theta$, the graph of $w_{\Theta}$ consists of the initial conditions whose trajectories remain bounded for all time, and forms the boundary between the initial points that are attracted to $y=-\infty$ and those attracted to $y=+\infty$. Thus Theorem 1 says that this 
fractal basin boundary almost surely has Hausdorff dimension equal to its boxcounting dimension $D$. This result supports the conjecture that Hausdorff and box-counting dimension typically coincide for attractors [6] and more generally for sets of dynamical interest.

Many prior results have been obtained on the Hausdorff dimension of the graphs of functions of the form

$$
f(x)=\sum_{n=0}^{\infty} b_{n}^{D-2} g\left(b_{n} x+\theta_{n}\right),
$$

where $1<D<2$, the function $g$ is periodic (and usually assumed to be Lipschitz continuous) and the sequence $\left\{b_{n}\right\}$ satisfies $b_{n+1} \geq \rho b_{n}$ for some $\rho>1$. Besicovitch and Ursell [4] consider the case of the "sawtooth function" $g(x)$, defined to be the distance from $x$ to the nearest integer, and $\theta_{n}=0$. They show that the Hausdorff dimension of the graph of $f$ can take on any prescribed value between 1 and $D$ for an appropriate sequence $\left\{b_{n}\right\}$ that grows faster than exponentially as $n \rightarrow \infty$; on the other hand, they prove that if $b_{n+1} / b_{n} \rightarrow \infty$ sufficiently slowly as $n \rightarrow \infty$, then the graph of $f$ must have Hausdorff dimension $D$.

Subsequent papers have generally concentrated on the case $b_{n}=b^{n}$ for some $b>1$, as in the original Weierstrass function; then

$$
f(x)=\sum_{n=0}^{\infty} b^{(D-2) n} g\left(b^{n} x+\theta_{n}\right),
$$

and it is expected that the Hausdorff dimension of the graph of $f$ is typically equal to $D$. In [11], it is proved that if $\theta_{n}=0$ and $g$ is $C^{1}$ and periodic (or almost periodic), then either $f$ is $C^{1}$ or the graph of $f$ has box-counting dimension $D$. Notice that if $\theta_{n}=0$ then $f$ is $C^{1}$ if and only if

$$
g(x)=h(x)-b^{D-2} h(b x)
$$

for some $C^{1}$ periodic function $h(x)$; in this case $f(x)=h(x)$. Bedford [1] proves a formula for the box-counting dimension of more general graphs that arise in dynamical systems. Rezakhanlou [19] shows for $\theta_{n}=0$ and $g(x)=\cos x$ that the packing dimension of the graph of $f$ is $D$. However, cases in which the Hausdorff dimension can be proved to equal $D$ have been elusive. Mauldin and Williams [16] obtain a lower bound of the form $D-C / \log b$ on the Hausdorff dimension of the graph of $f$ for arbitrary $\theta_{n}$, and a general class of functions $g$. Przytycki and Urbanski [18] obtain a different lower bound which is not as strong for large $b$ but establishes that the Hausdorff dimension of the graph of $f$ is greater than 1 for the (non-smooth) functions $f$ considered in [11].

There are a number of papers $[12,2,23,24]$ that give general support to the conjecture [1] that for a function that is (in some sense) self-affine, the box-counting and Hausdorff dimensions of its graph coincide if and only if its "occupation measure" is absolutely continuous with respect to Lebesgue measure. (Given a function $h$, the occupation measure $\mu_{h}$ of a set $S$ is defined to be the Lebesgue measure of $\{x: h(x) \in S$.) Most of the results in this direction require a notion of self-affinity that excludes the Weierstrass function, but a result of Kôno [12] implies that for $\theta_{n}=0$, integer $b$, and $a b$ enough larger than 1 , if the occupation measure $\mu_{f}$ has a bounded density function, then the Hausdorff dimension of the graph of $f$ is $D$. Pitt [17] is able to show that if the terms in the sum defining $w(x)$ are each multiplied by suitable independent, identically distributed random coefficients, then 
almost surely the occupation measure $\mu_{w}$ has an $L^{2}$ density function (and thus in particular is absolutely continuous); this offers another approach to randomizing $w(x)$ so that the Hausdorff dimension of its graph should almost surely be $D$.

In [18] it is also shown that if $\theta_{n}=0, b=2$, and $g$ is a Rademacher function $(g(x)=-1$ if $j \leq x<j+1 / 2$ and $g(x)=1$ if $j-1 / 2 \leq x<j$ for integer $j)$, then the Hausdorff dimension of the graph of $f$ is equal to $D$ if the occupation measure $\mu_{f}$ is absolutely continuous with respect to Lebesgue measure (this was also proved with an additional hypothesis in [20] and [9]), but is strictly less than $D$ for certain values of $D$ for which $\mu_{f}$ is singular. Ledrappier [13] proves that the Hausdorff dimension of the graph of $f$ is $D$ when $\theta_{n}=0$ and $g$ is a sawtooth function (defined above), provided $D$ avoids certain values. In both of these cases, the exceptional values of $D$ are related to the set $S$ of numbers $\lambda \in(1 / 2,1)$ for which the probability distribution of the random series $\sum \pm \lambda^{n}$, where the signs are chosen independently with probability $1 / 2$ for each sign, fails to be absolutely continuous with respect to Lebesgue measure. The set $S$ is known to be nonempty, and it is a long-standing problem of Erdös to further describe $S$ and its complement. It was recently proved by Solomyak [21] that $S$ has Lebesgue measure zero. Thus it is now known that when $g$ is a sawtooth function and $\theta_{n}=0$, the Hausdorff dimension of the graph of $f$ is equal to $D$ for almost every $D \in(1,2)$.

Brief discussions of the problem of the Hausdorff dimension of the graph of the Weierstrass function, along with additional references, can be found in the books of Falconer [5] and Mattila [15].

Most of the results obtained after [4] depend on the precise exponential nature of the frequencies $b^{n}$. In contrast, Theorem 1 can be extended to apply to functions of the form

$$
f_{\Theta}(x)=\sum_{n=0}^{\infty} a_{n} g\left(b_{n} x+\theta_{n}\right),
$$

where the frequencies $b_{n}$ and amplitudes $a_{n}$ need only exhibit an approximate exponential dependence on $n$. The precise hypotheses are given in Theorem 2 of Section 4, which concludes that the Hausdorff dimension of the graph of $f_{\Theta}$ is $D$ for almost every sequence $\Theta=\left\{\theta_{0}, \theta_{1}, \ldots\right\}$; this holds even if $D$ takes on one of the values that is exceptional in the results described above. The function $g$ need only be Lipschitz and periodic, and satisfy an additional technical condition which holds, for instance, if $g$ is real analytic. This extra condition on $g$ rules out the sawtooth function; on the other hand, it does not rule out the example described above which produces a smooth $f_{\Theta}$ when $\theta_{n}=0$ for each $n$. Thus it is not possible to improve Theorem 2 to a result about every sequence $\Theta$ without some additional restriction on $g$.

\section{Definitions And Notation}

The usual definition of the Hausdorff dimension of a Borel set $A \in \mathbf{R}^{n}$ is as follows. For $s \geq 0$ and $\delta>0$, define

$$
H_{\delta}^{s}(A)=\inf \sum_{i}\left|U_{i}\right|^{s}
$$

where $|U|=\sup \{|x-y|: x, y \in U\}$ denotes the diameter of a nonempty set $U$ and the infimum is taken over all countable collections $\left\{U_{i}\right\}$ of sets for which $A \subset \bigcup_{i} U_{i}$ 
and $\left|U_{i}\right| \leq \delta$. As $\delta$ decreases, $H_{\delta}^{s}(A)$ cannot decrease, and therefore it has a limit (possibly infinite) as $\delta \rightarrow 0$; define

$$
H^{s}(A)=\lim _{\delta \rightarrow 0} H_{\delta}^{s}(A) .
$$

The quantity $H^{s}(A)$ is known as the $s$-dimensional Hausdorff measure of $A$. It can be shown that for a given $A$ there is a value $\operatorname{dim}(A)$ for which $H^{s}(A)=\infty$ for $s<\operatorname{dim}(A)$ and $H^{s}(A)=0$ for $s>\operatorname{dim}(A)$. The Hausdorff dimension $\operatorname{dim}(A)$ is defined to be this value; that is,

$$
\operatorname{dim}(A)=\sup \left\{s: H^{s}(A)=\infty\right\}=\inf \left\{s: H^{s}(A)=0\right\} .
$$

The above definition is useful in bounding the Hausdorff dimension of a set $A$ from above. An upper bound on $H^{s}(A)$ can be obtained by examining specific covers of $A$ by sets $U_{i}$ of small diameter, and if $H^{s}(A)$ is shown to be finite for some $s$, then $\operatorname{dim}(A) \leq s$. However, lower bounds are more difficult to obtain directly because all possible covers $\left\{U_{i}\right\}$ must be considered.

Another definition of Hausdorff dimension can be given in terms of the $t$-energy $I_{t}(\mu)$ of a Borel measure $\mu$ supported on $A$, defined by

$$
I_{t}(\mu)=\iint \frac{d \mu(x) d \mu(y)}{|x-y|^{t}} .
$$

It can be proved (see [5] or [15], for instance) that if $t>\operatorname{dim}(A)$, then $I_{t}(\mu)=\infty$ for all measures $\mu$ supported on $A$, whereas if $t<\operatorname{dim}(A)$, there exists a measure $\mu$ supported on $A$ such that $I_{t}(\mu)<\infty$. Thus we can also write

$$
\operatorname{dim}(A)=\sup \left\{t: I_{t}(\mu)<\infty \text { for some measure } \mu \text { supported on } A\right\} .
$$

A lower bound $t$ on $\operatorname{dim}(A)$ can be obtained by constructing a measure $\mu$ supported on $A$ for which $I_{t}(\mu)$ is finite. We mention in passing that for fixed $\mu$, the quantity $\sup \left\{t: I_{t}(\mu)<\infty\right\}$ is often called the correlation dimension of $\mu$, and the above relation states that the Hausdorff dimension of $A$ is the supremum over all measures $\mu$ supported on $A$ of the correlation dimension of $\mu$.

\section{Proof of the main Result}

In this section we prove Theorem 1, keeping in mind that the argument can be generalized considerably. In the next section we state the more general result as Theorem 2, and discuss its proof.

Proof of Theorem 1. Let $H=[0,1]^{\infty}$, endowed with the uniform probability measure, and let $\Theta=\left\{\theta_{0}, \theta_{1}, \ldots\right\}$ denote a point in $H$. Recall that $D=2+\log a / \log b$, or in other words $a=b^{D-2}$, so we can write

$$
w_{\Theta}(x)=\sum_{n=0}^{\infty} b^{(D-2) n} \cos \left(2 \pi\left(b^{n} x+\theta_{n}\right)\right) .
$$

The goal is to prove that for almost every $\Theta \in H$, the Hausdorff dimension of the graph of $w_{\Theta}$ is $D$. The fact that the Hausdorff dimension of the graph of $w_{\Theta}$ is at most $D$ for all $\Theta \in H$ is well known, but for completeness we prove it here. 
We consider the graph of $w_{\Theta}$ over a finite interval $J$. We begin with an estimate which shows that $w_{\Theta}$ is Hölder continuous on $J$ with exponent $2-D$. For $x, y \in J$,

$$
\begin{aligned}
\left|w_{\Theta}(x)-w_{\Theta}(y)\right| & \leq \sum_{n=0}^{\infty} b^{(D-2) n}\left|\cos \left(2 \pi\left(b^{n} x+\theta_{n}\right)\right)-\cos \left(2 \pi\left(b^{n} y+\theta_{n}\right)\right)\right| \\
& \leq \sum_{n=0}^{\infty} b^{(D-2) n} \min \left(2,2 \pi b^{n}|x-y|\right) \\
& \leq \sum_{n=0}^{m-1} 2 \pi b^{(D-1) n}|x-y|+\sum_{n=m}^{\infty} 2 b^{(D-2) n} \\
& =2 \pi \frac{b^{(D-1) m}-1}{b^{D-1}-1}|x-y|+2 \frac{b^{(D-2) m}}{1-b^{D-2}}
\end{aligned}
$$

for all $m>0$. If $|x-y| \leq 1$, let $m$ be the positive integer with $b^{-m}<|x-y| \leq$ $b^{-(m-1)}$. Since $1<D<2$ it follows that

$$
\begin{aligned}
\left|w_{\Theta}(x)-w_{\Theta}(y)\right| & \leq 2 \pi \frac{(b /|x-y|)^{D-1}}{b^{D-1}-1}|x-y|+2 \frac{|x-y|^{2-D}}{1-b^{D-2}} \\
& \leq\left(\frac{2 \pi b^{D-1}}{b^{D-1}-1}+\frac{2}{1-b^{D-2}}\right)|x-y|^{2-D} .
\end{aligned}
$$

Let $L$ be the length of $J$, and let $N>L$ be a positive integer. Divide $J$ into $N$ intervals of equal length. By the above estimate, on a given interval of length $L / N$ the values of $w_{t} h$ vary by at most $C(L / N)^{2-D}$, with $C$ independent of $N$; thus the graph of $w_{t} h$ over this interval can be covered by at most $C(L / N)^{1-D}+1$ squares of length $L / N$. Then the graph of $w_{\Theta}$ over all of $J$ can be covered by at most $N\left(C(L / N)^{1-D}+1\right)=C L^{1-D} N^{D}+N$ squares of length $L / N$. It follows that the $D$-dimensional Hausdorff measure of the graph of $w_{\Theta}$ over $J$ is finite, and the Hausdorff dimension of the graph is at most $D$.

We turn now to the proof that the Hausdorff dimension of the graph of $w_{\Theta}$ over $J$ is at least $D$ for almost every $\Theta \in H$. Let $\mu_{\Theta}$ be the measure supported on the graph of $w_{\Theta}$ that is induced by Lebesgue measure $\nu$ on $J$. That is, for $S \subset \mathbf{R}^{2}$,

$$
\mu_{\Theta}(S)=\nu\left(\left\{x \in J:\left(x, w_{\Theta}(x)\right) \in S\right\}\right) .
$$

Then the $t$-energy of $\mu_{\Theta}$ is

$$
I_{t}\left(\mu_{\Theta}\right)=\int_{J} \int_{J} \frac{d x d y}{\left((x-y)^{2}+\left(w_{\Theta}(x)-w_{\Theta}(y)\right)^{2}\right)^{t / 2}} .
$$

We will prove for $t<D$ that $I_{t}\left(\mu_{\Theta}\right)$ is finite for almost every $\Theta \in H$, which implies that the Hausdorff dimension of the graph of $w_{\Theta}$ is at least $t$. Choosing a sequence of values of $t$ approaching $D$, we conclude that for almost every $\Theta \in H$, the Hausdorff dimension of the graph of $w_{\Theta}$ is at least $D$.

Fix $t \in(1, D)$. We will show that the integral

$$
E_{t}=\int_{H} I_{t}\left(\mu_{\Theta}\right) d \Theta
$$

is finite; it then follows that the integrand is finite for almost every $\Theta$. By the Tonelli theorem,

$$
E_{t}=\int_{J} \int_{J} \int_{H} \frac{d \Theta}{\left((x-y)^{2}+\left(w_{\Theta}(x)-w_{\Theta}(y)\right)^{2}\right)^{t / 2}} d x d y .
$$


We claim that there exists $C>0$ such that for $0<|x-y| \leq 1 /\left(2 b^{2}\right)$,

$$
\int_{H} \frac{d \Theta}{\left((x-y)^{2}+\left(w_{\Theta}(x)-w_{\Theta}(y)\right)^{2}\right)^{t / 2}} \leq C|x-y|^{D-t-1}
$$

Since $t<D$, it follows that $E_{t}<\infty$.

Fix $x$ and $y$ with $0<|x-y| \leq 1 /\left(2 b^{2}\right)$, and let $z=w_{\Theta}(x)-w_{\Theta}(y)$. Regarding $z$ as a function of the random sequence $\Theta$, we will show that $z$ has a bounded density function $h(z)$. (Of course $h$ depends on $x$ and $y$.) It follows that

$$
\begin{aligned}
\int_{H} \frac{d \Theta}{\left((x-y)^{2}+\left(w_{\Theta}(x)-w_{\Theta}(y)\right)^{2}\right)^{t / 2}} & =\int_{-\infty}^{\infty} \frac{h(z) d z}{\left((x-y)^{2}+z^{2}\right)^{t / 2}} \\
& =\int_{-\infty}^{\infty} \frac{h(|x-y| w)|x-y| d w}{|x-y|^{t}\left(1+w^{2}\right)^{t / 2}} \\
& \left.\leq \sup _{z} h(z)\right)|x-y|^{1-t} \int_{-\infty}^{\infty} \frac{d w}{\left(1+w^{2}\right)^{t / 2}}
\end{aligned}
$$

Thus to complete the proof we need only show that $h(z) \leq C^{\prime}|x-y|^{D-2}$ for some $C^{\prime}>0$ that is independent of $x$ and $y$.

Now

$$
\begin{aligned}
z & =w_{\Theta}(x)-w_{\Theta}(y) \\
& =\sum_{n=0}^{\infty} b^{(D-2) n}\left(\cos \left(2 \pi\left(b^{n} x+\theta_{n}\right)\right)-\cos \left(2 \pi\left(b^{n} y+\theta_{n}\right)\right)\right) \\
& =\sum_{n=0}^{\infty} 2 b^{(D-2) n} \sin \left(2 \pi b^{n}(y-x) / 2\right) \sin \left(2 \pi\left(b^{n}(x+y) / 2+\theta_{n}\right)\right) \\
& =\sum_{n=0}^{\infty} q_{n} \sin \left(r_{n}+2 \pi \theta_{n}\right),
\end{aligned}
$$

where $q_{n}$ and $r_{n}$ do not depend on $\Theta$ for all $n$. Let $z_{n}=q_{n} \sin \left(r_{n}+2 \pi \theta_{n}\right)$; then $z_{0}, z_{1}, \ldots$ are independent random variables (since $\theta_{0}, \theta_{1}, \ldots$ are independent) with density functions

$$
h_{n}\left(z_{n}\right)=\left\{\begin{array}{cc}
\frac{1}{\sqrt{q_{n}^{2}-z_{n}^{2}},} & \left|z_{n}\right|<\left|q_{n}\right|, \\
0, & \left|z_{n}\right| \geq\left|q_{n}\right|
\end{array}\right.
$$

(since $r_{n}+2 \pi \theta_{n}$ is uniformly distributed on an interval of length $2 \pi$ ). It follows that the density function $h(z)$ for $z=z_{0}+z_{1}+\ldots$ is the infinite convolution $h_{0} * h_{1} * \cdots$. Since the maximum value of a probability density cannot increase under convolution with another probability density, any upper bound we obtain on a finite convolution $h_{j} * \cdots * h_{k}$ is an upper bound on $h(z)$ as well.

Next, recall that

$$
q_{n}=2 b^{(D-2) n} \sin \left(2 \pi b^{n}(y-x) / 2\right)
$$

and that $0<|x-y|<1 /\left(2 b^{2}\right)$. Let $k \geq 2$ be the integer for which $(1 / 2) b^{-k-1}<$ $|x-y| \leq(1 / 2) b^{-k}$. Then

$$
\frac{\pi}{2 b^{3}}<\left|2 \pi b^{k-2} \frac{y-x}{2}\right|<\left|2 \pi b^{k} \frac{y-x}{2}\right| \leq \frac{\pi}{2}
$$

and hence

$$
\left|q_{n}\right|>2 \sin \left(\frac{\pi}{2 b^{3}}\right) b^{(D-2) k}>2 \sin \left(\frac{\pi}{2 b^{3}}\right)|x-y|^{2-D}
$$


for $n=k-2, k-1, k$. Let $\|\cdot\|_{p}$ denote the $L^{p}$ norm, and notice that $h_{n} \in L^{p}$ for $p<2$. It follows that for $n=k-2, k-1, k$,

$$
\left\|h_{n}\right\|_{\frac{3}{2}}=K\left|q_{n}\right|^{-1 / 3} \leq K^{\prime}|x-y|^{(D-2) / 3},
$$

where $K$ is an absolute constant and $K^{\prime}$ depends only on $b$. By Young's inequality (see $\S \mathrm{V} .1$ of [22], for instance),

$$
\left\|h_{k-1} * h_{k}\right\|_{3} \leq\left\|h_{k-1}\right\|_{\frac{3}{2}}\left\|h_{k}\right\|_{\frac{3}{2}}
$$

and then by Hölder's inequality,

$$
\begin{aligned}
h_{k-2} * h_{k-1} * h_{k}(z) & \leq\left\|h_{k-2}\right\|_{\frac{3}{2}}\left\|h_{k-1} * h_{k}\right\|_{3} \\
& \leq\left\|h_{k-2}\right\|_{\frac{3}{2}}\left\|h_{k-1}\right\|_{\frac{3}{2}}\|h\|_{\frac{3}{2}} \leq K^{\prime 3}|x-y|^{D-2} .
\end{aligned}
$$

The same bound then applies to $h(z)$, and the proof is complete.

\section{A more general Result}

Let

$$
f_{\Theta}(x)=\sum_{n=0}^{\infty} a_{n} g\left(b_{n} x+\theta_{n}\right),
$$

where $g$ is Lipschitz and periodic with period $1,\left\{a_{n}\right\}$ is positive and summable, $\left\{b_{n}\right\}$ is positive and increasing, and $\Theta=\left\{\theta_{0}, \theta_{1}, \ldots\right\} \in H$.

Theorem 2. Assume the following:

(i) $\rho b_{n} \leq b_{n+1} \leq \sigma b_{n}$ for some $\sigma>\rho>1$ and all $n$;

(ii) $\lim _{n \rightarrow \infty}\left(\log a_{n} / \log b_{n}\right)=D-2$ for some $1<D<2$;

(iii) there exist a positive integer $p$ and constants $0<\ell<1$ and $M>0$ such that for all $\ell / \sigma^{p} \leq \delta \leq \ell$ the density function of $g(x+\delta)-g(x)$, where $x$ is chosen randomly according to a uniform distribution on $[0,1]$, has $L^{p /(p-1)}$ norm at most $M$.

Then for almost every $\Theta \in H$, the Hausdorff dimension of the graph of $f_{\Theta}$ is $D$.

Notice that if $g$ is $C^{\infty}$, condition (iii) holds as long as $g(x+\delta)-g(x)$ does not have critical points of arbitrarily high (or infinite) order. Singularities in the density for the values of this function occur only when the function has derivative zero; a quadratic critical point requires only that $p>2$, a cubic critical point requires $p>3$, and so on. If $g$ is real analytic, then $g(x+\delta)-g(x)$ meets this condition as long as $\delta$ is restricted to a closed interval that does not contain a multiple of the period of $g$. Even if less smoothness is assumed for $g$, it should be possible to show that condition (iii) holds for "generic" $g$.

Proof of Theorem 2 (sketch). The proof is very similar to that of Theorem 1, and we will just point out the differences here. For the upper bound on the dimension, we require only that $g$ is Lipschitz, $b_{n+1} \geq \rho b_{n}$, and that for all $\varepsilon>0$, by condition (ii), $a_{n} \leq b_{n}^{D-2+\varepsilon}$ for $n$ sufficiently large. Then by the same argument as in the proof of Theorem 1, the Hausdorff dimension of the graph of $f_{\Theta}$ is at most $D+\varepsilon$, and letting $\varepsilon \rightarrow 0$ we have the desired upper bound $D$.

Likewise for all $\varepsilon>0$, we have for $n$ sufficiently large that $a_{n} \geq b_{n}^{D-2-\varepsilon}$. For a given $t \in(1, D)$ we choose $\varepsilon$ so that $t<D-\varepsilon$, and the proof is just like that of Theorem 1 once we show that for $|x-y|$ sufficiently small, $z=f_{\Theta}(x)-f_{\Theta}(y)$ has a density function $h(z) \leq C^{\prime}|x-y|^{D-2-\varepsilon}$. Now $z=z_{0}+z_{1}+\cdots$, where 
$z_{n}=a_{n}\left(g\left(b_{n} x+\theta_{n}\right)-g\left(b_{n} y+\theta_{n}\right)\right)$, and condition (iii) ensures that $z_{n}$ has a density function $h_{n} \in L^{p /(p-1)}$ for $\ell / \sigma^{p} \leq b_{n}|x-y| \leq \ell$. Condition (i) ensures that at least $p$ values of $n$ meet this condition, and Young's and Hölder's inequalities imply that $h(z)$ is bounded above by the product of the $L^{p /(p-1)}$ norms of $p$ of these $h_{n}$. A simple scaling argument shows that for such $n$ the $L^{p /(p-1)}$ norm of $h_{n}$ is bounded by a constant (independent of $x$ and $y$ ) times $a_{n}^{-1 / p}$, and the proof is complete since $a_{n} \geq b_{n}^{D-2-\varepsilon} \geq(\ell /|x-y|)^{D-2-\varepsilon}$ for every such $n$.

In the case $b_{n}=b^{n}$ for integer $b$, the graph of $f_{\Theta}$ can be thought of as a fractal basin boundary for the iteration of random maps that are a generalization of the maps $T_{\theta}$ discussed in Section 1. In this case the cosine is replaced by $g$, and the vertical stretching factor $1 / a$ can, like the phase $\theta$, be chosen randomly with respect to some prescribed probability distribution. This distribution need only satisfy the condition that $|\log (1 / a)|$ has a finite mean value and that $\log (1 / a)$ has mean $M \in(0, \log b)$, whence by a formulation of the strong law of large numbers due to Kolmogorov (see p. 261 in [7]), condition (ii) is met almost surely with $D=2-M / \log b$.

\section{ACKNOWLEDGMENTS}

The author was supported by the National Science Foundation (Divisions of Mathematical and Physical Sciences) and by the U.S. Department of Energy (Mathematical, Information, and Computational Sciences Division, High Performance Computing and Communications Program). The author is grateful to D. Mauldin for a very careful reading of an early version of this manuscript, and to J. Yorke for his suggestions.

\section{REFERENCES}

1. T. Bedford, The box dimension of self-affine graphs and repellers, Nonlinearity 2 (1989), 53-71. MR 90e:58091

2. T. Bedford \& M. Urbanski, The box and Hausdorff dimension of self-affine sets, Ergod. Th. Dynam. Sys. 10 (1990), 627-644. MR 92b:58125

3. M. V. Berry and Z. V. Lewis, On the Weierstrass-Mandelbrot fractal function, Proc. Royal Soc. Lond. A 370 (1980), 459-484. MR 81d:00005

4. A. S. Besicovitch and H. D. Ursell, Sets of fractional dimensions (V): On dimensional numbers of some continuous curves, J. London Math. Soc. (2) 32 (1937), 142-153.

5. K. J. Falconer, Fractal Geometry: Mathematical Foundations and Applications, New York: J. Wiley \& Sons (1990). MR 92j:28008

6. J. D. Farmer, E. Ott, and J. A. Yorke, The dimension of chaotic attractors, Physica 7D (1983), 153-180. MR 84m:58022

7. B. V. Gnedenko, The Theory of Probability, 4th ed., New York: Chelsea (1967). MR 36:913

8. G. H. Hardy, Weierstrass's non-differentiable function, Trans. Amer. Math. Soc. 17 (1916), 301-325.

9. T.-Y. Hu \& K.-S. Lau, The sum of Rademacher functions and Hausdorff dimension, Math. Proc. Camb. Phil. Soc. 108 (1990), 97-103. MR 91d:28020

10. B. R. Hunt \& J. A. Yorke, Smooth dynamics on Weierstrass nowhere differentiable curves, Trans. Amer. Math. Soc. 325 (1991), 141-154. MR 91h:58071

11. J. L. Kaplan, J. Mallet-Paret, and J. A. Yorke, The Lyapunov dimension of a nowhere differentiable attracting torus, Ergod. Th. Dynam. Sys. 4 (1984), 261-281. MR 86h:58091

12. N. Kôno, On self-affine functions, Japan J. Appl. Math. 3 (1986), 259-269. MR 88i:26013

13. F. Ledrappier, On the dimension of some graphs, Contemp. Math. 135 (1992), 285-293. MR 94d:28007

14. B. B. Mandelbrot, Fractals: Form, Chance and Dimension, San Francisco: Freeman (1977). MR 57:11224 
15. P. Mattila, Geometry of Sets and Measures in Euclidean Spaces, Cambridge Univ. Press (1995). MR 96h:28006

16. R. D. Mauldin and S. C. Williams, On the Hausdorff dimension of some graphs, Trans. Amer. Math. Soc. 298 (1986), 793-804. MR 88c:28006

17. Loren Pitt, private communication.

18. F. Przytycki and M. Urbanski, On the Hausdorff dimension of some fractal sets, Studia Math. 93 (1989), 155-186. MR 90f: 28006

19. F. Rezakhanlou, The packing measure of the graphs and level sets of certain continuous functions, Math. Proc. Camb. Phil. Soc. 104 (1988), 347-360. MR 89m:28006

20. Y. Shiota and T. Sekiguchi, Hausdorff dimension of graphs of some Rademacher series, Japan J. Appl. Math. 7 (1990), 121-129. MR 91e:28009

21. B. Solomyak, On the random series $\sum \pm \lambda^{n}$ (an Erdös problem), Ann. Math. 142 (1995), 611-625. MR 97d:11125

22. E. M. Stein and G. Weiss, Introduction to Fourier Analysis on Euclidean Spaces, Princeton Univ. Press (1971). MR 46:4102

23. M. Urbanski, The probability distribution and Hausdorff dimension of self-affine functions, Probab. Th. Rel. Fields 84 (1990), 377-391. MR 91e:58103

24. M. Urbanski, The Hausdorff dimension of the graphs of continuous self-affine functions, Proc. Amer. Math. Soc. 108 (1990), 921-930. MR 90g:26004

Institute for Physical Science and Technology, University of Maryland, College PARK, MARYLAND 20742-2431

E-mail address: bhunt@ipst.umd.edu 